\title{
Natural Transform along with HPM Technique for Solving Fractional ADE
}

\author{
N. Pareek $\left(\mathbb{D},{ }^{1}\right.$ A. Gupta ${ }^{(D)},{ }^{2}$ G. Agarwal $\left(\mathbb{D},{ }^{3}\right.$ and D. L. Suthar $\mathbb{D}^{4}$ \\ ${ }^{1}$ Department of Mathematics, Bhagat Singh Govt. P.G. College, Jaora, India \\ ${ }^{2}$ Department of Mathematics, Govt. M.V.M, Bhopal, India \\ ${ }^{3}$ Department of Mathematics and Statistics, Manipal University Jaipur, Rajasthan, India \\ ${ }^{4}$ Department of Mathematics, Wollo University, P.O. Box: 1145, Dessie, Ethiopia
}

Correspondence should be addressed to D. L. Suthar; dlsuthar@gmail.com

Received 16 April 2021; Accepted 8 July 2021; Published 28 July 2021

Academic Editor: Younes Menni

Copyright (C) 2021 N. Pareek et al. This is an open access article distributed under the Creative Commons Attribution License, which permits unrestricted use, distribution, and reproduction in any medium, provided the original work is properly cited.

\begin{abstract}
The authors of this paper solve the fractional space-time advection-dispersion equation (ADE). In the advection-dispersion process, the solute movement being nonlocal in nature and the velocity of fluid flow being nonuniform, it leads to form a heterogeneous system which approaches to model the same by means of a fractional ADE which generalizes the classical ADE, where the time derivative is substituted through the Caputo fractional derivative. For the study of such fractional models, various numerical techniques are used by the researchers but the nonlocality of the fractional derivative causes high computational expenses and complex calculations so the challenge is to use an efficient method which involves less computation and high accuracy in solving such models numerically. Here, in order to get the FADE solved in the form of convergent infinite series, a novel method NHPM (natural homotopy perturbation method) is applied which couples Natural transform along with the homotopy perturbation method. The homotopy peturbation method has been applied in mathematical physics to solve many initial value problems expressed in the form of PDEs. Also, the HPM has an advantage over the other methods that it does not require any discretization of the domains, is independent of any physical parameters, and only uses an embedding parameter $p \in[0,1]$. The HPM combined with the Natural transform leads to rapidly convergent series solutions with less computation. The efficacy of the used method is shown by working out some examples for time-fractional ADE with various initial conditions using the NHPM. The Mittag-Leffler function is used to solve the fractional space-time advection-dispersion problem, and the impact of changing the fractional parameter $\alpha$ on the solute concentration is shown for all the cases.
\end{abstract}

\section{Introduction and Preliminaries}

Fractional calculus generalizes the integration and differentiation of integer order to arbitrary order that is being studied for the past 300 years. The growing interest of researchers in this field has led to solving the real-world issues in type of fractional differential equations due to their nonlocal behavior, and these equations are well suited to describe various phenomena in the field of engineering and science. Also, fractional derivatives are capable to model various processes mathematically which exhibit the memory and hereditary properties [1-5].
The ADE arises in the study of transport of solute or Brownian motion of particles in a fluid occurring due to the simultaneous occurrence of advection and particle dispersion. Fractional advection-dispersion equation describes the phenomena of anomalous diffusion of the particles in the transport process in a better way; as in anomalous diffusion, the solute transport is quicker or speedier than time's inferred square root given by Baeumer et al. [6]. The equation is used to study groundwater pollution, pollution of the atmosphere produced by smoke or dust, the spread of chemical solutes and contaminant discharges, etc. [7]. Hence, FADE has attracted the attention of many researchers. 
Hence, the interest of the researchers lies in solving the FADE to find out the solute concentration at a particular instant of time and space. Analytical solution of onedimensional ADE was found by Jaiswal et al. [8]. Huang et al. [9] solve the one-dimensional fractional flux ADE and found the finite element solutions. The intermediate fractional ADE was studied by El-Sayed et al. [10]. To solve the space-time fractional ADE, Momani and Odibat [7] utilized the $\mathrm{ADM}$ and variational iteration approach. In this continuation, Yildirim and Koçak [11] solve the space-time fractional ADE by applying homotopy perturbation technique in Caputo sense and Hikal and Abu Ibrahim [12] solved it by the Adomian decomposition method. Alliche and Chikh [13] studied the nonpremixed chaotic fire of the hydrogen-air downward injector system using the generalized finite rate chemistry model. Liu et al. [14] applied numerical methods to study various advection-dispersion models. Rocca et al. [15] developed a general solution to the fractional diffusion-advection equation for solar cosmic-ray transport. Ramani et al. [16] explored the fractional decreased differential transform approach for revisiting the analytical-approximate formulation of the timefractional Rosenau-Hyman problem. The extended differential transform approach was used by Garg and Manohar [17] to solve the space-time fractional Fokker Planck (FFP) equation analytically. Also, Habenom et al. [18] studied the formulation of FFP equation using fractional power series technique. The N-transform was used by Khan and Khan [19] to study the unsteady fluid flow over a plane wall, and $\mathrm{N}$-transform of some functions along with the properties was presented. Belgacem and Silambarasan [20] renamed it as Natural transform which they used to solve Bessel's differential equation with a polynomial coefficient and also Maxwell's equation.

In this article, first, we recall few concepts of fractional calculus, Natural transform, and HPM which have been used in our main findings, in Sections 2, 3, and 4, respectively. Then, we gave a solution to the space-time ADE by the NHPM in Section 5, and at the last, Section 6 contains some related examples, which show the efficiency of this method. In Section 7, a conclusion has been discussed.

\section{Basic Definitions}

The Riemann-Liouville and Caputo-type fractional integral operator and its properties are discussed in this section. These definitions and properties (see detail [1-3]) will be used to get the main results.

Definition 1. Let $\mathfrak{f}(x)$ with $x>0$ be a real-valued function. If there is a real number $p>\mu$, it is said to be in the space $\mathscr{C}$ in $R$. Such that $\mathfrak{f}(x)=x^{p} \mathfrak{f}_{1} x$, where $\mathfrak{f}_{1}(x) \in \mathscr{C}[0, \infty)$.

Definition 2. Let $\mathfrak{f}(x)$ with $x>0$ be a real-valued function; then, it would seem to be in space $\mathscr{C}_{\mu}^{n}, m \in N \cup\{0\}$, if $\mathfrak{f}^{(m)} \in$ $\mathscr{C}_{\mu}$.
Definition 3. For a function $\mathfrak{f} \in C_{\mu}$, where $\mu=1$, the R-L fractional integral operator of order $\vartheta=0$ is described as

$$
\begin{gathered}
\rrbracket^{\vartheta} \mathfrak{f}(z)=\frac{1}{\Gamma(\vartheta)} \int_{0}^{z}(z-t)^{\vartheta-1} \tilde{\mathfrak{f}}(t) d t ; \vartheta>0, z>0, \\
\rrbracket^{0} \mathfrak{f}(z)=\mathfrak{f}(z), \\
\rrbracket^{\vartheta} \rrbracket^{\vartheta} \mathfrak{f}(z)=\rrbracket^{\vartheta+\vartheta} \mathfrak{f}(z), \\
\rrbracket^{\vartheta} z^{\vartheta}=\frac{\Gamma(\vartheta+1)}{\Gamma(\vartheta+\vartheta+1)} z^{\vartheta+\vartheta} .
\end{gathered}
$$

Definition 4. In Caputo's view, the fractional derivative of $\mathfrak{f} \in \mathscr{C}^{n-1}$ is expressed by

$$
D^{9} \mathfrak{f}(z)= \begin{cases}\frac{1}{\Gamma(n-\vartheta)} \int_{0}^{z}(z-\tau)^{n-9-1} \mathfrak{f}^{n}(\tau) d \tau, & n-1<\vartheta<n, n \in N^{*}, \\ \frac{d^{n}}{d t^{n}} \mathfrak{f}(z), & \vartheta=n .\end{cases}
$$

Also,

$$
\begin{gathered}
D^{\vartheta} \mathscr{K}=0 ; \mathscr{K} \text { being a constant, } \\
D^{\vartheta} z^{\vartheta}= \begin{cases}\frac{\Gamma(\vartheta+1)}{\Gamma(\vartheta-\vartheta+1)} z^{\vartheta-\vartheta}, & \vartheta>\vartheta-1, \\
0, & \vartheta=\vartheta-1 .\end{cases}
\end{gathered}
$$

Definition 5. The two-parameter M-L function is described as follows:

$$
E_{\vartheta, \vartheta}(z)=\sum_{k=0}^{\infty} \frac{z^{k}}{\Gamma(\vartheta k+\vartheta)}
$$

Consequently, the one-parameter $\mathrm{M}-\mathrm{L}$ function is described as follows:

$$
E_{\vartheta}(z)=E_{\vartheta, 1}(z)=\sum_{k=0}^{\infty} \frac{z^{k}}{\Gamma(\vartheta k+1)}
$$

\section{Natural Transform}

Over the set, Natural transform is specified:

$$
A=\left\{\mathrm{f}(t): \exists M, \tau_{1}, \tau_{2}|v(t)|<M e^{|t| / \tau_{j}}, \text { if } t \in(-1)^{j} \times[0, \infty)\right\},
$$

$\mathbb{N}[\mathrm{f}(t)]=F(s, u)=\frac{1}{u} \int_{0}^{\infty} e^{-s t / u} \mathrm{f}(t) d t ; \quad u>0, s>0, t \in(0, \infty)$

where $s$ and $u$ denote the Natural transform variables [21, 22].

Remark 6.

(i) If $u=1,(7)$ reduces to the Laplace transform 
TABLE 1: Relation between $\mathbb{N}$-transforms, Sumudu transform, and Laplace transform.

\begin{tabular}{lccc}
\hline$f(t)$ & $\mathbb{N}[f(t)]$ & $\mathbb{S}[f(t)]$ & $\mathbb{L}[f(t)]$ \\
\hline 1 & $\frac{1}{s}$ & 1 & $\frac{1}{s}$ \\
$t$ & $\frac{u}{s^{2}}$ & $u$ & $\frac{1}{s^{2}}$ \\
$e^{a t}$ & $\frac{1}{s-a u}$ & $\frac{1}{1-a u}$ & $\frac{1}{s-a}$ \\
$\frac{t^{n-1}}{n-1 !}, n=1,2 .$. & $\frac{u^{n-1}}{s^{n}}$ & $u^{n-1}$ & $\frac{1}{s^{n}}$ \\
$\sin (t)$ & $\frac{u}{s^{2}+u^{2}}$ & $\frac{u}{1+u^{2}}$ & $\frac{1}{1+s^{2}}$ \\
\hline
\end{tabular}

TABle 2: Properties of $\mathbb{N}$-transforms.

\begin{tabular}{lc}
\hline Function & Natural transform of the function \\
\hline$f(t)$ & $F(s, u)$ \\
$f(a t)$ & $\frac{1}{a} F(s, u)$ \\
$f^{\prime}(t)$ & $\frac{s}{u} F(s, u)-\frac{1}{u} f(0)$ \\
$f^{\prime \prime}(t)$ & $\frac{s^{2}}{u^{2}} F(s, u)-\frac{s}{u^{2}} f(0)-\frac{1}{u} f^{\prime}(0)$ \\
$a f(t) \pm b g(t)$ & $a F(s, u) \pm b G(s, u)$ \\
\hline
\end{tabular}

(ii) If $s=1$, (7) reduces to the Sumudu transform

$\mathbb{N}$-transforms of some elementary functions and the conversions to Sumudu and Laplace [19, 21-23] are given in Tables 1 and 2.

\section{The Homotopy Perturbation Method}

The general form of the time-dependent differential equation (see [24-26]) can be written as

$$
\mathbb{A}(u(\varsigma, t))-f(\varsigma, t)=0,
$$

where $\mathbb{A}$ is the differential operator, $u(\varsigma, t)$ is the unknown function, $r$ is the independent variables for space, $t$ is the independent variables for time, and $f(\varsigma, t)$ is the analytic function.

In general, $\mathbb{A}$ can be divided into $\mathbb{L}$ (linear) and $\mathbb{N}$ (nonlinear) component s.t.:

$$
\mathbb{A}=\mathbb{L}+\mathbb{N} .
$$

By substituting the value of $A$ in (8),

$$
\mathbb{L}(u(\varsigma, t))+\mathbb{N}(u(\varsigma, t))-f(\varsigma, t)=0 .
$$

Using the homotopy technique presented by Liao [27], a homotopy $\varnothing(r, t: p)$ can be constructed which satisfies

$$
\begin{aligned}
\mathbb{H}(\varnothing(\varsigma, t: p), p)= & (1-p)\left\{\mathbb{L}(\varnothing(\varsigma, t: p))-\mathbb{L}\left(u_{0}(\varsigma, t)\right)\right\} \\
& +p\{\mathbb{A}(\varnothing(\varsigma, t: p))-f(\varsigma, t)\}=0,
\end{aligned}
$$

where $p \in[0,1]$ is an embedding parameter and $u_{0}(r, t)$ is an initial guess for $u(r, t)$ satisfying initial/boundary conditions. The homotopy equation (11) can be written in an equivalent form as

$$
\begin{aligned}
& \mathbb{L}(\varnothing(\varsigma, t: p))-\mathbb{L}\left(u_{0}(\varsigma, t)\right)+p\{N(\varnothing(\varsigma, t: p)) \\
& \left.\quad+\mathbb{L}\left(u_{0}(\varsigma, t)\right)-f(\varsigma, t)\right\}=0 .
\end{aligned}
$$

Hence, when $p=0$, we obtain

$\mathbb{H}(\varnothing(\varsigma, t: 0), 0)=\mathbb{L}(\varnothing(\varsigma, t: 0))-\mathbb{L}\left(u_{0}(\varsigma, t)\right)=0$,

and when $p=1$, we get

$$
\mathbb{H}(\varnothing(\varsigma, t: 1), 1)=\mathbb{A}(\varnothing(\varsigma, t: 1))-f(\varsigma, t)=0 .
$$

We observe that $u(\varsigma, t)$ is the solution of (14) as well as (8) and if $\mathbb{L}$ is taken to be linear, $u_{0}(\varsigma, t)$ is the only solution of (13). So, we have

$$
\begin{aligned}
& \varnothing(\varsigma, t: 0)=u_{0}(\varsigma, t), \\
& \varnothing(\varsigma, t: 1)=u(\varsigma, t) .
\end{aligned}
$$

Change in $p$ from 0 to 1 is followed by change in $\varnothing(\varsigma, t: p)$ from $u_{0}(\varsigma, t)$ to $u(\varsigma, t)$, termed as deformation. If the embedding parameter $p(1 \geq p \geq 0)$ is thought to be tiny, according to the classic perturbation technique, the solution to the given equation may be assumed as a power series in $p$, so

$$
\varnothing(\varsigma, t: p)=u_{0}(\varsigma, t)+p u_{1}(\varsigma, t)+p^{2} u_{2}(\varsigma,)+\cdots,
$$

for $p=1$,

$$
u(\varsigma, t)=u_{0}(\varsigma, t)+u_{1}(\varsigma, t)+u_{2}(\varsigma, t)+\cdots,
$$

which gives the approximate solution of (8). The series in (17) converges in most of the cases and leads to the exact solution.

\section{Solution of the Space-Time ADE by the NHPM}

The classical one-dimensional ADE with constant parameters is of the form (see [14])

$$
\frac{\partial C}{\partial \tau}=D \frac{\partial^{2} C}{\partial \xi^{2}}-V \frac{\partial C}{\partial \xi},
$$

where $V$ is the drift velocity, $\xi$ is the spatial coordinate, $D>0$ is the constant diffusivity, and $C(\xi, \tau)$ is the solute concentration. 
To write equation (18) in a simplified form by setting $t=\tau V, x=\xi$ and replacing $C(\xi, \tau)$ by $C(x, t)$, it reduced into

$$
C_{t}=\mu C_{x x}-C_{x}
$$

where $\mu=D / V$.

We write the general form of the space-time fractional $\mathrm{ADE}$ as

$$
D_{t}^{9} C=\mu D_{x}^{p 9} C-D_{x}^{r 9} C,
$$

with $\vartheta=1 / m, 0<\vartheta \leq 1,1<p \vartheta \leq 2,0<r \vartheta \leq 1, m, p, r \varepsilon N, 0<x$ $<L, D_{x}^{p 9}=D_{x}^{9} D_{x}^{9} \cdots D_{x}^{9}$ (p times), $D_{x}^{r 9}=D_{x}^{9} D_{x}^{9} \cdots D_{x}^{9}(r$ times $)$, $D_{t}^{\alpha}$ and $D_{x}^{9}$ are the Caputo fractional derivatives.

The initial condition is

$$
C(x, 0)=f(x) .
$$

$\mathbb{N}$-transform of (20) is written as

$$
\frac{s^{9}}{u^{9}} C(x, s, u)-\frac{s^{9-1}}{u^{9}} C(x, 0)=\mathbb{N}^{+}\left[\mu D_{x}^{p \vartheta} C-D_{x}^{r 9} C\right] .
$$

Now, by substituting initial condition from (21) in the above equation, we obtain

$$
C(x, s, u)=\frac{f(x)}{s}+\frac{u^{9}}{s^{9}} \mathbb{N}^{+}\left[\mu D_{x}^{p 9} C-D_{x}^{r 9} C\right] .
$$

Applying the inverse $\mathbb{N}$-transform on (23), we get

$$
C(x, t)=f(x)+\mathbb{N}^{-1}\left[\frac{u^{9}}{s^{9}} \mathbb{N}^{+}\left\{\mu D_{x}^{p 9} C-D_{x}^{r 9} C\right\}\right] .
$$

By using the homotopy perturbation method, we can write

$$
C(x, t)=\sum_{n=0}^{\infty} p^{n} C_{n}(x, t) .
$$

Substituting (25) in (24),

$$
\begin{aligned}
& \sum_{n=0}^{\infty} p^{n} C_{n}(x, t)=f(x) \\
& \quad+p\left[\mathbb{N}^{-1}\left\{\frac{u^{9}}{s^{9}} \mathbb{N}^{+}\left(\sum_{n=0}^{\infty} \mu p^{n} D_{x}^{p 9} C_{n}-\sum_{n=0}^{\infty} p^{n} D_{x}^{r 9} C_{n}\right)\right\}\right] .
\end{aligned}
$$

Comparison of the coefficients of like powers of $p$ on both sides yields to the corresponding assumptions:

$$
p^{0}: C_{0}(x, t)=f(x)
$$

$$
\begin{aligned}
p^{1}: C_{1}(x, t) & =\mathbb{N}^{-1}\left\{\frac{u^{\vartheta}}{s^{9}} \mathbb{N}^{+}\left(\mu D_{x}^{p^{9}} C_{0}-D_{x}^{r 9} C_{0}\right)\right\} \\
& =\mathbb{N}^{-1}\left\{\frac{u^{9}}{s^{9}} \mathbb{N}^{+}\left(\mu D_{x}^{p 9} f(x)-D_{x}^{r 9} f(x)\right)\right\} \\
& =\left(\mu D_{x}^{p \vartheta} f(x)-D_{x}^{r 9} f(x)\right) \mathbb{N}^{-1}\left\{\frac{u^{9}}{s^{9+1}}\right\} \\
& =\frac{t^{9}}{\Gamma(\vartheta+1)}\left(\mu D_{x}^{p \vartheta}-D_{x}^{r \vartheta}\right) f(x),
\end{aligned}
$$

$$
\begin{aligned}
p^{2}: C_{2}(x, t)= & \mathbb{N}^{-1}\left\{\frac{u^{9}}{s^{9}} \mathbb{N}^{+}\left(\mu D_{x}^{p 9} C_{1}-D_{x}^{r 9} C_{1}\right)\right\} \\
= & \mathbb{N}^{-1}\left\{\frac { u ^ { 9 } } { s ^ { 9 } } \mathbb { N } ^ { + } \left(\mu D_{x}^{p 9}\left(\mu D_{x}^{p 9} f(x)-D_{x}^{r 9} f(x)\right) \frac{t^{9}}{\Gamma(9+1)}\right.\right. \\
& \left.\left.-D_{x}^{r 9}\left(\mu D_{x}^{p 9} f(x)-D_{x}^{r 9} f(x)\right) \frac{t^{9}}{\Gamma(9+1)}\right)\right\} \\
= & \left(\mu^{2} D_{x}^{2 p 9} f(x)-2 \mu D_{x}^{(p+r) 9} f(x)+D_{x}^{2 r 9} f(x)\right) \\
& \times \mathbb{N}^{-1}\left\{\frac{u^{9}}{s^{9}} \mathbb{N}^{+}\left(\frac{t^{9}}{\Gamma(\vartheta+1)}\right)\right\} \\
= & \left(\mu^{2} D_{x}^{2 p 9} f(x)-2 \mu D_{x}^{(p+r) 9} f(x)+D_{x}^{2 r 9} f(x)\right) \mathbb{N}^{-1}\left\{\frac{u^{29}}{s^{29+1}}\right\} \\
= & \frac{t^{29}}{\Gamma(29+1)}\left(\mu D_{x}^{p 9}-D_{x}^{r 9}\right)^{2} f(x) .
\end{aligned}
$$

Similarly,

$$
p^{3}: C_{3}(x, t)=\frac{t^{39}}{\Gamma(3 \vartheta+1)}\left(\mu D_{x}^{p 9}-D_{x}^{r 9}\right)^{3} f(x)
$$

and so on.

The analytic series solution of (20) can be given as

$$
C(x, t)=\lim _{N \longrightarrow \infty} \sum_{n=0}^{N} C_{n}(x, t),
$$

$$
\begin{aligned}
C(x, t)= & C_{0}(x, t)+C_{1}(x, t)+C_{2}(x, t)+C_{3}(x, t)+\cdots \\
= & f(x)+\frac{t^{\alpha}}{\Gamma(\vartheta+1)}\left(\mu D_{x}^{p 9}-D_{x}^{r 9}\right) f(x)+\frac{t^{2 \alpha}}{\Gamma(2 \vartheta+1)} \\
& \cdot\left(\mu D_{x}^{p 9}-D_{x}^{r 9}\right)^{2} f(x)+\frac{t^{3 \alpha}}{\Gamma(39+1)}\left(\mu D_{x}^{p 9}-D_{x}^{r 9}\right)^{3} f(x) \cdots \\
= & \left\{1+\frac{t^{\alpha}}{\Gamma(\vartheta+1)}\left(\mu D_{x}^{p 9}-D_{x}^{r 9}\right)+\frac{t^{2 \alpha}}{\Gamma(2 \vartheta+1)}\left(\mu D_{x}^{p 9}-D_{x}^{r 9}\right)^{2}\right. \\
& \left.+\frac{t^{3 \alpha}}{\Gamma(3 \vartheta+1)}\left(\mu D_{x}^{p 9}-D_{x}^{r 9}\right)^{3}+\cdots\right\} f(x) \\
= & \sum_{n=0}^{\infty}\left\{\frac{t^{n \alpha}}{\Gamma(n \vartheta+1)}\left(\mu D_{x}^{p 9}-D_{x}^{r 9}\right)^{n}\right\} f(x),
\end{aligned}
$$




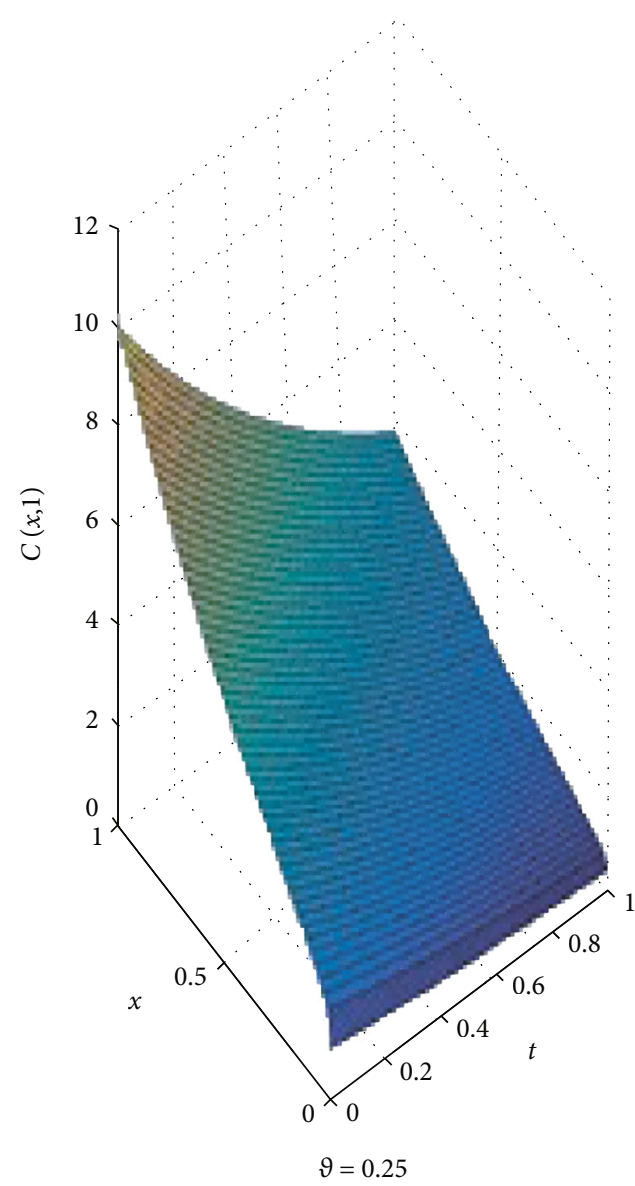

(a) $9=0.25$

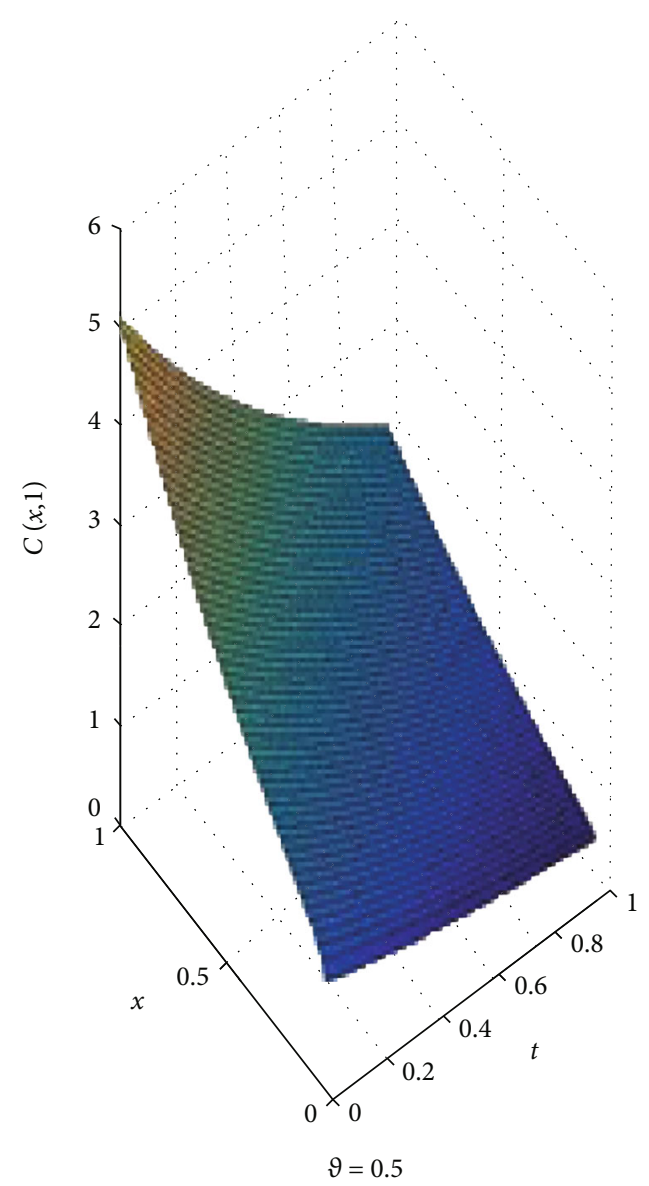

(b) $\vartheta=0.5$

Figure 1: Continued. 


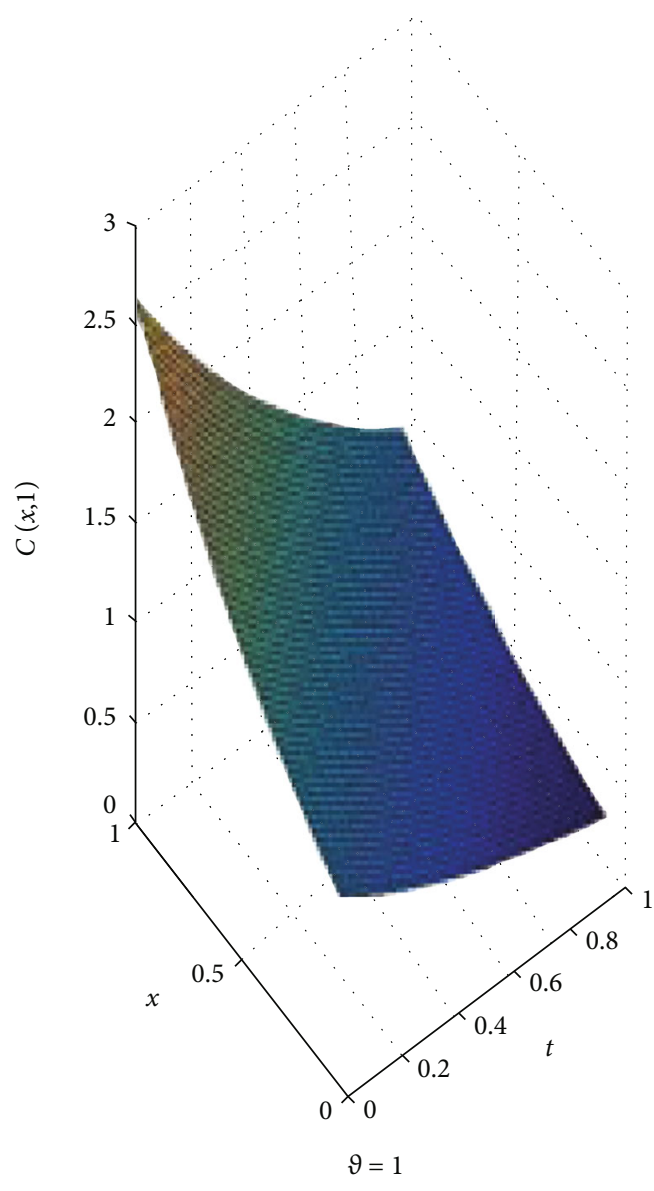

(c) $\vartheta=1$

Figure 1: Variation of the concentration $C(x, t)$ with the fractional parameter $\alpha$ for $\mu=.01$.

which can also be written as

$$
C(x, t)=\left[E_{\vartheta}\left\{t^{\vartheta}\left(\mu D_{x}^{p^{\vartheta}}-D_{x}^{r^{\vartheta}}\right)\right\}\right] f(x) .
$$

where $E_{\alpha}(x)$ is the one-parameter Mittag-Leffler function.

Remark 7. Setting $p=2, r=1$, (20) reduces to space-time fractional $\mathrm{ADE}$ of the form

$$
D_{t}^{9} C=\mu D_{x}^{29} C-D_{x}^{9} C
$$

and the solution is

$$
C(x, t)=\left[E_{\vartheta}\left\{t^{\vartheta}\left(\mu D_{x}^{2 \vartheta}-D_{x}^{9}\right)\right\}\right] f(x),
$$

if $f(x)=e^{-x}$, the solution is

$$
\begin{aligned}
C(x, t)= & {\left[E_{\vartheta}\left\{t^{\vartheta}\left(\mu D_{x}^{2 \vartheta}-D_{x}^{\vartheta}\right)\right\}\right] e^{-x}=\sum_{n=0}^{\infty} \frac{t^{n \vartheta}}{\Gamma(n \vartheta+1)} } \\
& \cdot \sum_{k=0}^{n}(-1)^{k} n_{c_{k}} \mu^{n-k} \sum_{s=\lfloor(2 n-k) \vartheta\rfloor}^{\infty} \frac{(-1)^{s} x^{s-(2 n-k) \vartheta}}{\Gamma(s-(2 n-k) \vartheta+1)},
\end{aligned}
$$

where $\lfloor$.$\rfloor denotes the ceiling function.$
This is the same as obtained by Hikal and Abu Ibrahim [12] using ADM.

\section{Examples}

Example 1. Consider the time-fractional ADE (setting $p=2$ , $r=1, m=1$ in $(20))$,

$$
D_{t}^{\alpha} C=\mu D_{x}^{2} C-D_{x} C,
$$

the initial condition being

$$
C(x, 0)=e^{-x} .
$$

Solution: by applying the NHPM,

$$
\begin{gathered}
p^{0}: C_{0}(x, t)=e^{-x} \\
p^{1}: C_{1}(x, t)=\mathbb{N}^{-1}\left\{\frac{u^{\vartheta}}{s^{\vartheta}} \mathbb{N}^{+}\left(\mu C_{0 x x}-C_{0 x}\right)\right\}=\frac{(\mu+1) t^{\vartheta}}{\Gamma(\vartheta+1)} e^{-x}, \\
p^{2}: C_{2}(x, t)=\mathbb{N}^{-1}\left\{\frac{u^{\vartheta}}{s^{\vartheta}} \mathbb{N}^{+}\left(\mu C_{1 x x}-C_{1 x}\right)\right\}=\frac{(\mu+1)^{2} t^{2 \vartheta}}{\Gamma(2 \vartheta+1)} e^{-x} .
\end{gathered}
$$




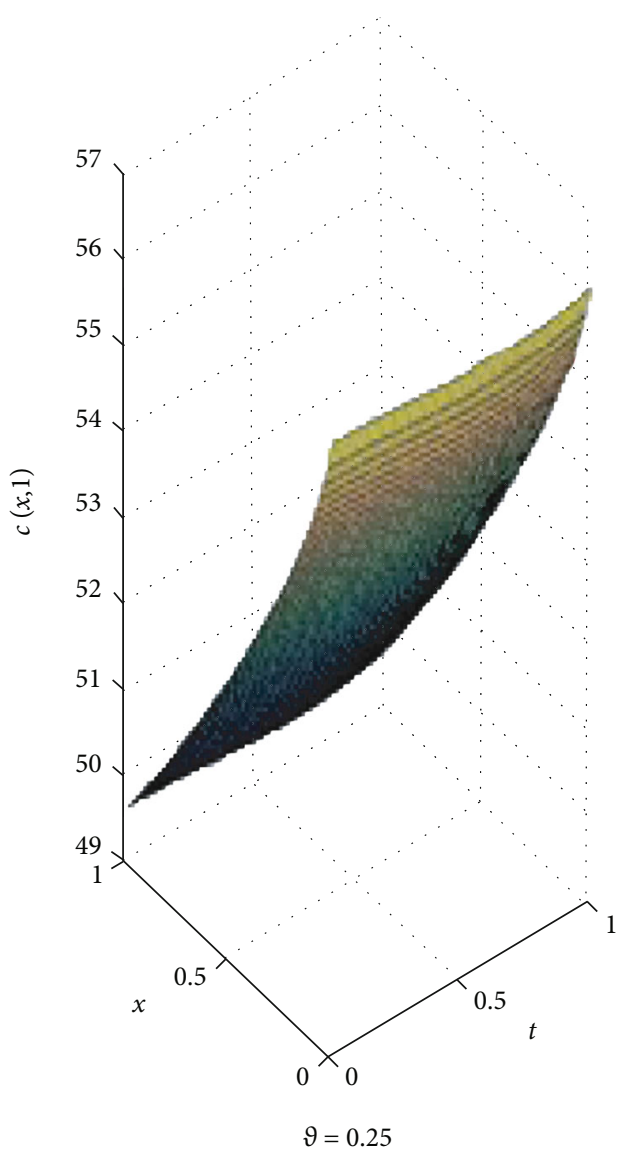

(a) $9=0.25$

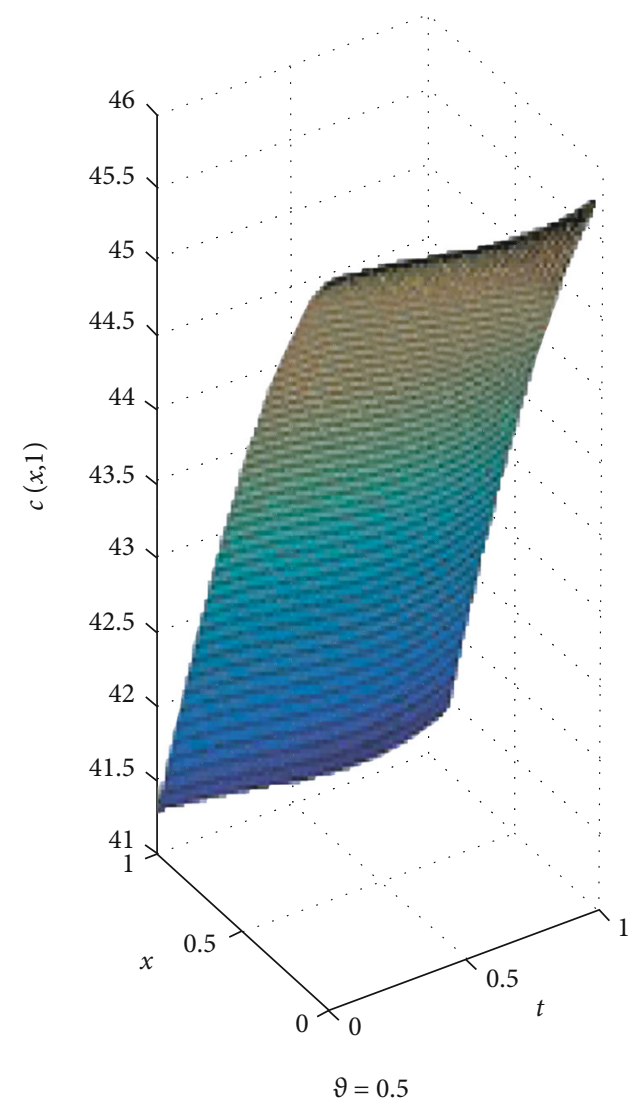

(b) $\vartheta=0.5$

Figure 2: Continued. 


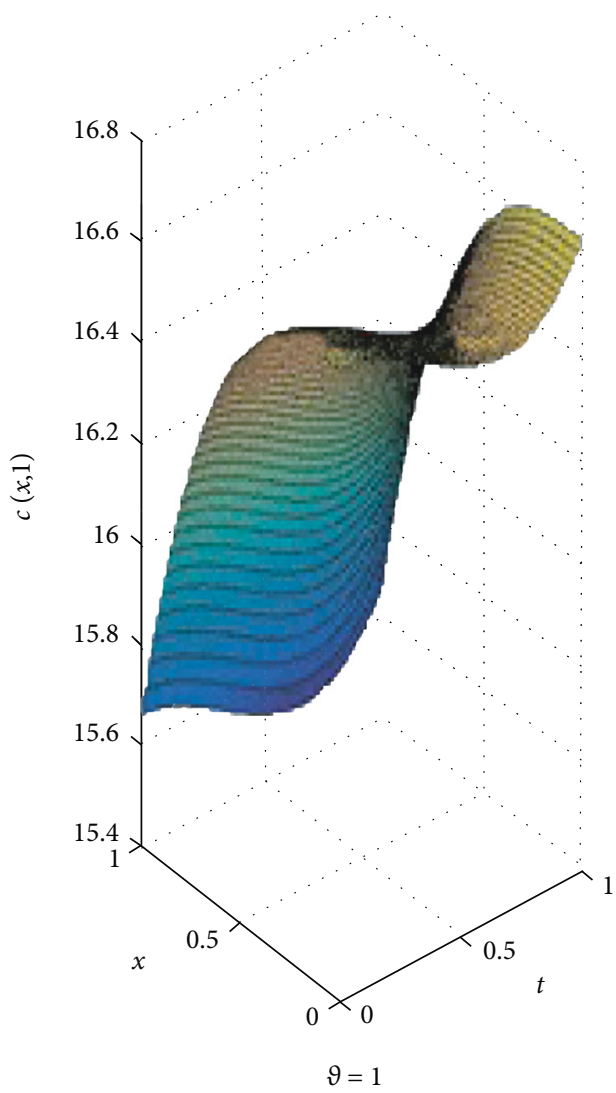

(c) $\vartheta=1$

Figure 2: Variation of the concentration $C(x, t)$ with the fractional parameter $\alpha$ for $\mu=.01$.

Similarly,

$$
p^{3}: C_{3}(x, t)=\frac{(\mu+1)^{3} t^{3 \vartheta}}{\Gamma(3 \vartheta+1)} e^{-x}
$$

and so on. Thus, the analytic series solution is given by

$$
\begin{aligned}
C(x, t) & =C_{0}(x, t)+C_{1}(x, t)+C_{2}(x, t)+C_{3}(x, t)+\cdots \\
& =e^{-x}\left\{1+\frac{(\mu+1) t^{\vartheta}}{\Gamma(\vartheta+1)}+\frac{(\mu+1)^{2} t^{2 \vartheta}}{\Gamma(2 \vartheta+1)}+\frac{(\mu+1)^{3} t^{3 \vartheta}}{\Gamma(3 \vartheta+1)}+\cdots\right\} \\
& =e^{-x} E_{\alpha}\left((1+\mu) t^{\vartheta}\right) .
\end{aligned}
$$

The solution converges to the exact solution of the ADE for $\alpha=1$ as obtained by El-Sayed et al. [10]:

$$
C(x, t)=e^{(1+\mu) t-x}
$$

The result obtained for Example 1 is presented in Figure 1.

Example 2. Equation (34) with the initial condition $C(x, 0)$ $=x^{3}-x^{2}$.
Solution: by applying the NHPM, we obtain

$$
\begin{gathered}
p^{0}: C_{0}(x, t)=x^{3}-x^{2} \\
p^{1}: C_{1}(x, t)=\left\{-3 x^{2}+2 x(1+3 \mu)-2 \mu\right\} \frac{t^{9}}{\Gamma(\vartheta+1)},
\end{gathered}
$$

$$
\begin{gathered}
p^{2}: C_{2}(x, t)=(6 x-2-12 \mu) \frac{t^{2 \vartheta}}{\Gamma(2 \vartheta+1)}, \\
p^{3}: C_{3}(x, t)=-6 \frac{t^{3 \vartheta}}{\Gamma(3 \vartheta+1)} \\
p^{4}: C_{4}(x, t)=0
\end{gathered}
$$

Thus, the analytic series solution is given by

$$
\begin{aligned}
C(x, t)= & C_{0}(x, t)+C_{1}(x, t)+C_{2}(x, t)+C_{3}(x, t)+\cdots C(x, t) \\
= & \left(x^{3}-x^{2}\right)+\left\{-3 x^{2}+2 x(1+3 \mu)-2 \mu\right\} \frac{t^{\vartheta}}{\Gamma(\vartheta+1)} \\
& +(6 x-2-12 \mu) \frac{t^{2 \vartheta}}{\Gamma(2 \vartheta+1)}-6 \frac{t^{3 \vartheta}}{\Gamma(3 \vartheta+1)} .
\end{aligned}
$$

The result obtained for Example 2 is presented in Figure 2. 


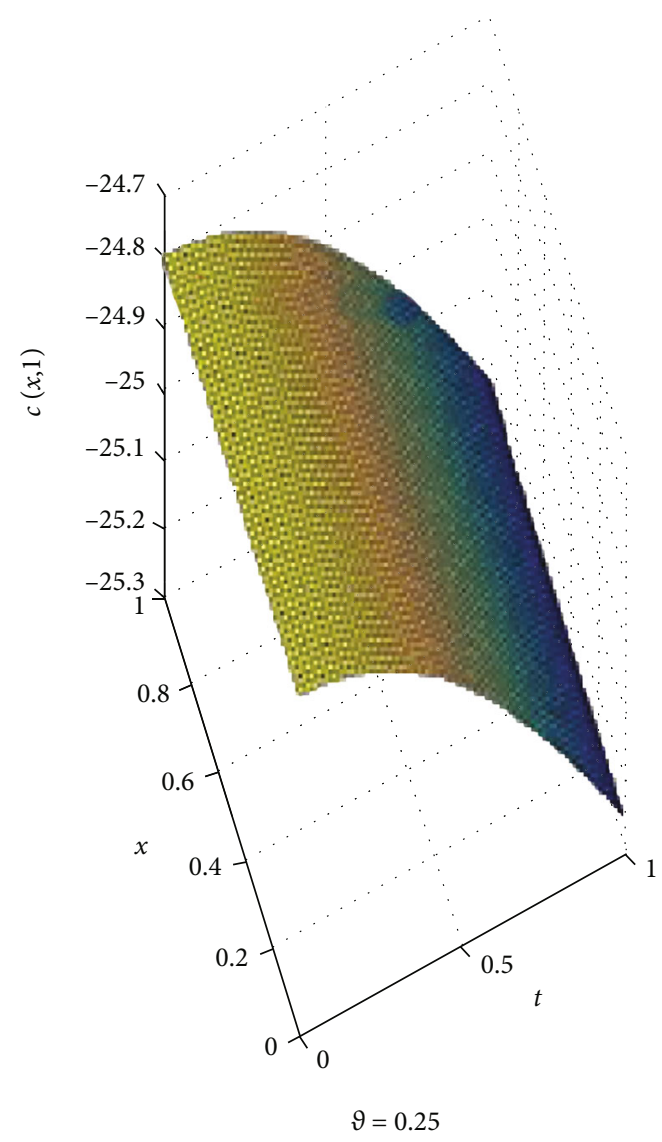

(a) $9=0.25$

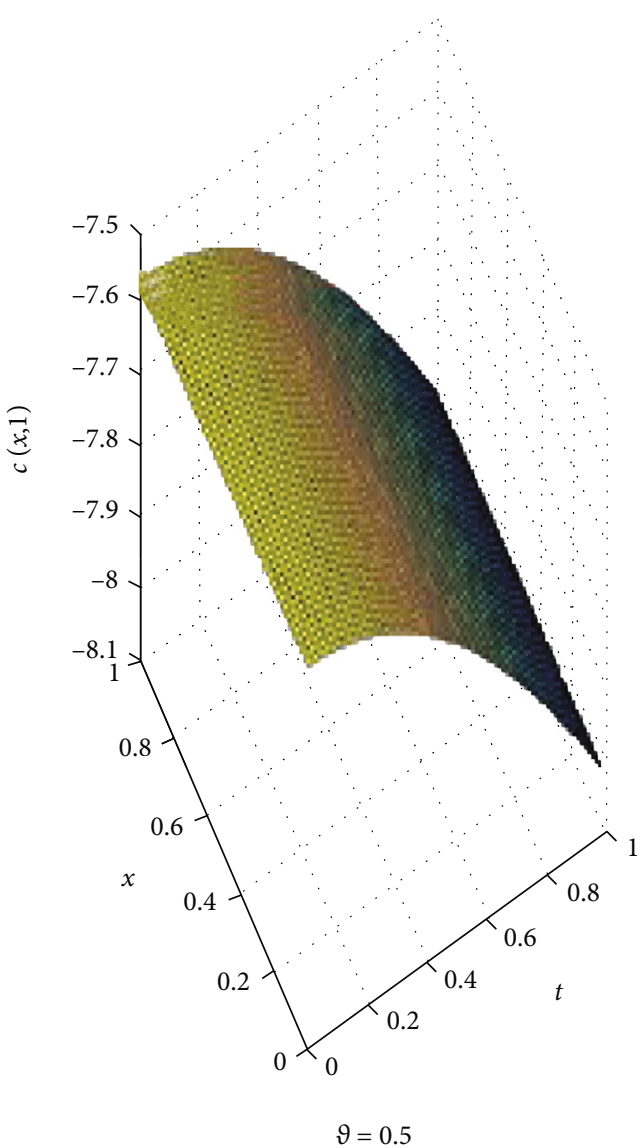

(b) $\vartheta=0.5$

Figure 3: Continued. 


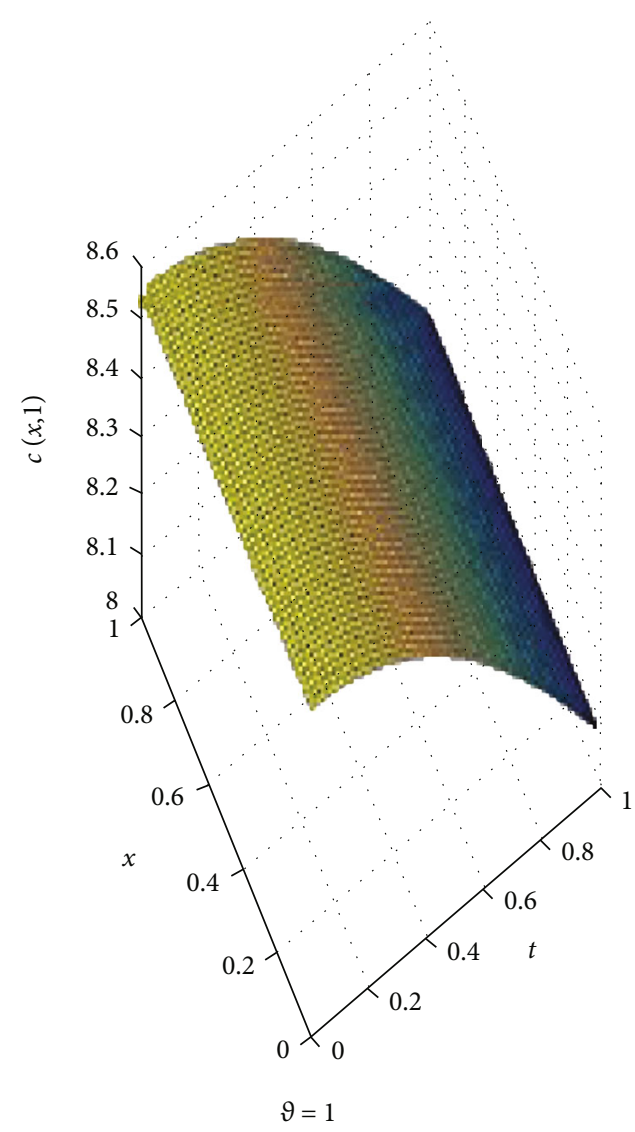

(c) $9=1$

Figure 3: Variation of the concentration $C(x, t)$ with the fractional parameter $\alpha$ for $\mu=.01$.

Example 3. Equation (34) with the initial condition $C(x, 0)$ $=\cos x$.

Solution: by applying the NHPM, we get

$$
\begin{gathered}
p^{0}: C_{0}(x, t)=\cos x \\
p^{1}: C_{1}(x, t)=(\sin x-\mu \cos x) \frac{t^{9}}{\Gamma(\vartheta+1)}, \\
\left.p^{2}: C_{2}(x, t)=\left(-\cos x-2 \mu \sin x+\mu^{2} \cos x\right)\right) \frac{t^{2 \vartheta}}{\Gamma(2 \vartheta+1)}, \\
p^{3}: C_{3}(x, t)=\left(-\sin x+3 \mu \cos x+3 \mu^{2} \sin x-\mu^{3} \cos x\right) \frac{t^{3 \vartheta}}{\Gamma(3 \vartheta+1)},
\end{gathered}
$$

and so on. Thus, the analytic series solution is given by

$$
\begin{aligned}
C(x, t)= & C_{0}(x, t)+C_{1}(x, t)+C_{2}(x, t)+C_{3}(x, t)+\cdots C(x, t) \\
= & \cos x+(\sin x-\mu \cos x) \frac{t^{\vartheta}}{\Gamma(\vartheta+1)} \\
& \left.+\left(-\cos x-2 \mu \sin x+\mu^{2} \cos x\right)\right) \frac{t^{29}}{\Gamma(2 \vartheta+1)} \\
& +\left(-\sin x+3 \mu \cos x+3 \mu^{2} \sin x-\mu^{3} \cos x\right) \\
& \cdot \frac{t^{39}}{\Gamma(3 \vartheta+1)}+\cdots .
\end{aligned}
$$

The result obtained for Example 3 is presented in Figure 3.

Remark 8 . The convergence of the series solutions obtained for the above cases can be proved by means of a comparison test using the Mittag-Leffler function.

\section{Conclusion}

In the present article, the NHPM is successfully applied to find the solution for the general form of the space-time fractional ADE and the analytic solution is found in terms of M$\mathrm{L}$ function for different cases. The results found show the dependence of solute concentration on the fractional order of the derivative along with the space and time variables. Solutions obtained for the three examples are plotted with spatial and time coordinate for different values of the fractional parameter $\alpha$ using MATLAB R2015a. Figure 1 exhibits a decrease in solute concentration corresponding to the increment in the fractional parameter $\alpha$. For the second case, the solution is in exact form and is plotted in Figure 2 showing that as the fractional parameter $\alpha$ increases, the solute concentration decreases, whereas it increases with the corresponding increment in $\alpha$ for the third cases as is shown in Figure 3. Hence, the NHPM is a powerful technique to solve various models in the form of linear or nonlinear partial differential equations appearing in the field of science and 
engineering. The approach should be expanded to solve the two or three dimensions of space-time fractional ADM.

\section{Nomenclature}

$C(\xi, \tau)$ : Solute concentration ( $\mathrm{mol} / \mathrm{kgw})$

$D>0$ : Constant diffusivity $\left(\mathrm{m}^{2} / \mathrm{sec}\right)$

$V: \quad$ Drift velocity $(\mathrm{m} / \mathrm{sec})$

$\xi: \quad$ Spatial coordinate $(\mathrm{m})$

$\tau: \quad$ Time $(\mathrm{sec})$.

\section{Data Availability}

No data were used to support this study.

\section{Conflicts of Interest}

There is no conflict of interest regarding the publication of this article.

\section{References}

[1] I. Podlubny, Fractional Differential Equations, Academic Press, San Diego, 1999.

[2] K. B. Oldham and J. Spanier, The Fractional Calculus, Academic Press, New York, 1974.

[3] K. S. Miller and B. Ross, An Introduction to the Fractional Calculus and Fractional Differential Equations, John Wiley\& Sons, New York, 1993.

[4] L. Mistry, A. M. Khan, D. L. Suthar, and D. Kumar, "A new numerical method to solve non-linear fractional differential equations," International Journal of Innovative Technology and Exploring Engineering, vol. 8, no. 12, pp. 1-6, 2019.

[5] P. Ramani, A. M. Khan, and D. L. Suthar, "Generalized differential transform method and its application to solve nonlinear partial differential equations with space and time fractional derivatives," International Journal of Scientific Research and Review, vol. 7, no. 2, pp. 1-9, 2019.

[6] B. Baeumer, D. A. Benson, and M. M. Meerschaert, "Advection and dispersion in time and space," Physica A, vol. 350, no. 2-4, pp. 245-262, 2005.

[7] S. Momani and Z. Odibat, "Numerical solutions of the spacetime fractional advection-dispersion equation," Numerical Methods for Partial Differential Equations, vol. 25, no. 5, pp. 1238-1259, 2008.

[8] D. K. Jaiswal, A. Kumar, and R. R. Yadav, "Analytical solution to the one-dimensional advection-diffusion equation with temporally dependent coefficients," Journal of Water Resource and Protection, vol. 3, no. 1, pp. 76-84, 2011.

[9] Q. Huang, G. Huang, and H. Zhan, "A finite element solution for the fractional advection-dispersion equation," Advances in Water Resources, vol. 31, no. 12, pp. 1578-1589, 2008.

[10] A. M. A. El-Sayed, S. H. Behiry, and W. E. Raslan, “Adomian's decomposition method for solving an intermediate fractional advection-dispersion equation," Computer and Mathematics with Applications, vol. 59, no. 5, pp. 1759-1765, 2010.

[11] A. Yildirim and H. Kocak, "Homotopy perturbation method for solving the space-time fractional advection- dispersion equation," Advances in Water Resources, vol. 32, no. 12, pp. 1711-1716, 2009.
[12] M. M. Hikal and M. A. Abu Ibrahim, "On Adomian's decomposition method for solving a fractional advection-dispersion equation," International Journal of Pure and Applied mathematics, vol. 104, no. 1, pp. 43-56, 2015.

[13] M. Alliche and S. Chikh, "Study of non-premixed turbulent flame of hydrogen/air downstream co-current injector," International Journal of Hydrogen Energy, vol. 43, no. 6, pp. 35773585, 2018.

[14] F. Liu, P. Zhuang, and K. Burrage, "Numerical methods and analysis for a class of fractional advection-dispersion models," Computers \& Mathematcs with Applications, vol. 64, no. 10, pp. 2990-3007, 2012.

[15] M. C. Rocca, A. R. Plastino, A. Plastino, and A. L. De Paoli, "General solution of a fractional diffusion-advection equation for solar cosmic-ray transport," Physica A, vol. 447, pp. 402410, 2016.

[16] P. Ramani, A. M. Khan, and D. L. Suthar, "Revisiting analytical-approximate solution of time fractional RosenauHyman equation via fractional reduced differential transform method," International Journal on Emerging Technologies, vol. 10, no. 2, pp. 403-409, 2019.

[17] M. Garg and P. Manohar, "Analytical solution of space-time fractional Fokker Planck equations by generalized differential transform method," Le Matematiche, vol. 66, no. 2, pp. 91$101,2011$.

[18] H. Habenom, D. L. Suthar, and M. Aychluh, "Solution of fractional Fokker Planck equation using fractional power series method," Journal of Science and Arts, vol. 48, no. 3, pp. 593600, 2019.

[19] Z. H. Khan and W. A. Khan, "N-transform properties and applications," NUST journal of engineering sciences, vol. 1, no. 1, pp. 127-133, 2008.

[20] F. B. M. Belgacem and R. Silambarasan, "Maxwell's equations solutions through the natural transform," Mathematics in Engineering, Science and Aerospace, vol. 3, no. 3, pp. 313$323,2012$.

[21] F. B. M. Belgacem and R. Silambarasan, "Theory of natural transform," Mathematical Engineering, Science and Aerospace, vol. 3, no. 1, pp. 100-124, 2012.

[22] F. B. M. Belgacem and R. Silambarasan, "Advances in the natural transform," in AIP Conference Proceedings; 1493 January 2012, pp. 106-110, USA, 2012.

[23] M. G. M. Hussain and F. B. M. Belgacem, “Transient solutions of Maxwell's equations based on Sumudu transform," Progress in Electromagnetic Research, vol. 74, pp. 273-289, 2007.

[24] H. Ji-Huan, "Homotopy perturbation technique," Computer Methods in Applied Mechanics and Engineering, vol. 178, pp. 257-262, 1999.

[25] H. Ji-Huan, "The homotopy perturbation method for nonlinear oscillators with discontinuities," Applied Mathematical Computation, vol. 151, pp. 287-292, 2004.

[26] H. Ji-Huan, "Recent development of the homotopy perturbation method," Topological Methods in Nonlinear Analysis, vol. 31, pp. 205-209, 2008.

[27] S. J. Liao, "On the homotopy analysis method for nonlinear problems," Applied Mathematics and Computation, vol. 147, pp. 499-513, 2007. 